\title{
Renaissance of base deficit for the initial assessment of trauma patients: a base deficit- based classification for hypovolemic shock developed on data from 16,305 patients derived from the TraumaRegister DGU ${ }^{\circledR}$
}

Manuel Mutschler ${ }^{1,2^{*}}$, Ulrike Nienaber ${ }^{3}$, Thomas Brockamp ${ }^{1}$, Arasch Wafaisade ${ }^{1}$, Tobias Fabian ${ }^{1}$, Thomas Paffrath$^{1}$, Bertil Bouillon ${ }^{1}$, Marc Maegele ${ }^{1}$ and the TraumaRegister DGU ${ }^{4}$

See related commentary by Privette et al., http://ccforum.com/content/17/2/124

\begin{abstract}
Introduction: The recognition and management of hypovolemic shock still remain an important task during initial trauma assessment. Recently, we have questioned the validity of the Advanced Trauma Life Support (ATLS) classification of hypovolemic shock by demonstrating that the suggested combination of heart rate, systolic blood pressure and Glasgow Coma Scale displays substantial deficits in reflecting clinical reality. The aim of this study was to introduce and validate a new classification of hypovolemic shock based upon base deficit (BD) at emergency department (ED) arrival.
\end{abstract}

Methods: Between 2002 and 2010, 16,305 patients were retrieved from the TraumaRegister DGU ${ }^{\circledR}$ database, classified into four strata of worsening BD [class I (BD $\leq 2 \mathrm{mmol} / \mathrm{l})$, class II (BD $>2.0$ to $6.0 \mathrm{mmol} / \mathrm{l})$, class III (BD > 6.0 to $10 \mathrm{mmol} / \mathrm{l})$ and class $\mathrm{IV}(\mathrm{BD}>10 \mathrm{mmol} / \mathrm{/})$ ] and assessed for demographics, injury characteristics, transfusion requirements and fluid resuscitation. This new BD-based classification was validated to the current ATLS classification of hypovolemic shock.

Results: With worsening of $\mathrm{BD}$, injury severity score (ISS) increased in a step-wise pattern from $19.1( \pm 11.9)$ in class I to 36.7 ( \pm 17.6) in class IV, while mortality increased in parallel from $7.4 \%$ to $51.5 \%$. Decreasing hemoglobin and prothrombin ratios as well as the amount of transfusions and fluid resuscitation paralleled the increasing frequency of hypovolemic shock within the four classes. The number of blood units transfused increased from $1.5( \pm 5.9)$ in class I patients to $20.3( \pm 27.3)$ in class IV patients. Massive transfusion rates increased from $5 \%$ in class I to $52 \%$ in class IV. The new introduced BD-based classification of hypovolemic shock discriminated transfusion requirements, massive transfusion and mortality rates significantly better compared to the conventional ATLS classification of hypovolemic shock $(p<0.001)$.

Conclusions: BD may be superior to the current ATLS classification of hypovolemic shock in identifying the presence of hypovolemic shock and in risk stratifying patients in need of early blood product transfusion.

\footnotetext{
* Correspondence: manuelmutschler@web.de

'Department of Trauma and Orthopedic Surgery, Cologne-Merheim Medical Center (CMMC), University of Witten/Herdecke, Ostmerheimerstr. 200, D-

51109 Cologne, Germany

Full list of author information is available at the end of the article
} 


\section{Introduction}

The early recognition and management of hypovolemic shock in multiply injured patients are still among the most challenging tasks in the acute assessment and treatment of trauma patients. For the initial evaluation of circulatory depletion, the American College of Surgeons has defined in its training program 'Advanced Trauma Life Support' (ATLS) four classes of hypovolemic shock. This classification is based upon an estimated blood loss in percent together with corresponding vital signs [1,2]. For each class, ATLS allocates therapeutic recommendations (for example, the administration of intravenous fluids and blood products) [1]. Recently, the clinically validity of the ATLS classification of hypovolemic shock has been questioned by two analyses independently from each other on two large-scale trauma databases: the TARN (Trauma Audit and Research Network) registry and the TraumaRegister DGU ${ }^{\mathbb{B}}$, which had consisted of more than 140,000 trauma patients. According to both analyses, ATLS seems (a) to overestimate the degree of tachycardia associated with hypotension and (b) to underestimate mental disability in the presence of hypovolemic shock [3-5].

These observations and conclusions prompted us to develop an alternative approach for the early assessment of hypovolemic shock in the emergency department (ED). Several studies have already identified worsening base deficit (BD) as an indicator for increased transfusion requirement $[6,7]$. Furthermore, BD has been associated with increased mortality, intensive care unit (ICU) and in-hospital lengths of stay, and a higher incidence of shock-related complications such as acute respiratory distress syndrome, renal failure, hemocoagulative disorders, and multiorgan failure (MOF) [6-9]. Monitoring of BD has also been suggested as an indicator and monitoring parameter for the success of resuscitation efforts $[7,10,11]$. In times of point-of-care testing (POCT), BD can be assessed in a fast and easy manner and therefore is available within minutes after admission to the ED. The aim of this study was to introduce and validate a four-class BD-based classification of hypovolemic shock on datasets of severely injured patients derived from the TraumaRegister DGU ${ }^{\circledR}$ database.

\section{Materials and methods}

The TraumaRegister DGU ${ }^{\circledR}$

The TraumaRegister DGU ${ }^{\circledR}$ was founded in 1993 and details have been published in extenso elsewhere [3,12]. To date, datasets from approximately 70,000 patients from more than 450 hospitals have been entered into the database. The TraumaRegister DGU ${ }^{\circledR}$ captures all severe trauma patients, who either are admitted to the hospital via the ED with subsequent ICU/intermediate care (ICU/IMC) care or reach the hospital with vital signs and die prior to ICU/IMC admission. It was approved by the review board of the German Trauma Society (DGU) and is in compliance with the institutional requirements of its members.

\section{Data analyses}

In the present study, datasets of multiply injured patients entered into the TraumaRegister $D_{G}{ }^{\circledR}$ between 2002 and 2010 were analyzed. Inclusion criteria were age of at least 16 years, primary admission, and complete datasets for BD upon admission blood gas analysis as well as for systolic blood pressure (SBP), heart rate (HR), and Glasgow Coma Scale (GCS) score to rebuild the ATLS classification of hypovolemic shock for validation.

Characterization of the four classes of hypovolemic shock based upon base deficit at emergency department admission

According to Davis and colleagues [6], four different classes of shock were defined and analyzed. Class I ('no shock') was defined by a BD of not more than $2 \mathrm{mmol} /$ L, class II ('mild shock') by a BD of more than 2.0 to 6.0 $\mathrm{mmol} / \mathrm{L}$, class III ('moderate shock') by a BD of more than 6.0 to $10.0 \mathrm{mmol} / \mathrm{L}$, and class IV ('severe shock') by a $\mathrm{BD}$ of more than $10 \mathrm{mmol} / \mathrm{L}$. Each patient was allocated to the corresponding shock class I to IV according to BD upon ED arrival. Vital signs (for example, HR, SBP, and GCS score) were assessed as present upon ED arrival and at the scene of the accident. Shock index (SI), defined by the ratio of HR to SBP, was calculated for both time points. Further assessments included demographics and injury patterns as well as therapeutic interventions such as administration of blood products, intravenous fluids, and vasopressors. Massive transfusion (MT) was defined by the administration of at least 10 blood products between ED and ICU admission. Coagulopathy was defined by a Quick's value of not more than $70 \%$, which is equivalent to an international normalized ratio of approximately $1.3[13,14]$.

\section{Validation of the new base deficit-based classification to} the current ATLS classification of hypovolemic shock

For the validation of the new BD-based classification to the current ATLS classification of hypovolemic shock, the latter was interpreted as previously described [3]. Briefly, SBP, HR, and GCS score were assessed to allocate the patients into the respective ATLS groups of hypovolemic shock but with some minor modifications [3]. As stated above, allocation of patients into the respective classes of hypovolemic shock was limited if a combination of all three parameters was applied. Therefore, in the present analysis, we allocated each patient into the respective shock class I to IV by the vital sign (HR, SBP, or GCS score) that matches the criteria of the 
highest shock class. If patients had been intubated and mechanically ventilated prior to ED admission, the GCS score at the scene of injury was considered. Patients were classified according to their BD at ED admission and according to the criteria suggested by ATLS. Transfusion requirements as well as mortality rates within the four groups were compared.

\section{Statistical methods}

Data are presented as means \pm standard deviations for continuous variables or percentages for categorical variables. GCS scores are presented as medians and interquartile ranges. For continuous variables, normal distribution was excluded by using the Shapiro-Wilk test. To detect differences between the four groups of worsening BD, a Kruskal-Wallis test was performed. A Mann-Whitney $U$ test on pairwise comparisons was performed in case of a significant overall difference. Categorical variables were analyzed accordingly with the chisquare test. For all statistical analyses, a probability of less than 0.05 was considered to be statistically significant. All data were analyzed by using IBM SPSS 19 (IBM Corporation, Chicago, IL, USA).

\section{Results}

Characterization of the four classes of hypovolemic shock based upon base deficit at emergency department admission

In total, 16,305 patients were identified from the TraumaRegister DGU ${ }^{\mathbb{R}}$ for further analysis. General demographics and detailed information on injury severity, trauma mechanism, RISC (Revised Injury Severity Classification) prognosis, and outcome for the four classes of hypovolemic shock based upon BD at ED admission are shown in Table 1. Worsening of BD category was associated with increased injury severity and both increased morbidity and mortality. Consequently, ICU and overall in-hospital lengths of stay as well as times on ventilator were prolonged with worsening of BD category. Table 2 summarizes vital signs for the four classes of shock at the scene and upon ED admission. A significant increase in SI was observed through the groups I to IV. HR seemed unaltered within the four groups, and interestingly no group displayed a relevant tachycardia at all. A substantial hypotension with a mean SBP of $87 \pm 45$ $\mathrm{mm} \mathrm{Hg}$ was observed in patients with a BD of more than $10 \mathrm{mmol} / \mathrm{L}$ (class IV) only. GCS scores decreased from a median of 14 ( 3 to 15 ) in class I patients to 3 (3 to 3 ) in class IV patients, whereas the percentage of patients intubated and mechanically ventilated at the scene increased from $40.2 \%$ (class I) to $83.4 \%$ (class IV), respectively. Furthermore, hemoglobin levels dropped from $12.8 \pm 2.4 \mathrm{~g} / \mathrm{dL}$ (class I) to $9.1 \pm 3.3 \mathrm{~g} / \mathrm{dL}$ (class IV), and platelet counts declined substantially throughout the classes I to IV (Table 3). Coagulopathy, defined by a Quick's value of not more than $70 \%$, was found in patients with a BD of more than $6 \mathrm{mmol} / \mathrm{L}$ (classes III and IV).

An increase in BD category was associated with a progressively stepwise increasing number of blood products administered (Figure 1). On average, the number of blood units transfused increased from $1.5 \pm 5.9$ units in class I patients to $20.3 \pm 27.3$ units in class IV patients. Packed red blood cells were transfused most frequently, followed by fresh frozen plasma and platelet concentrates (Figure 1a). Simultaneously, observed and predicted transfusion requirements were concordant, as the number of blood products transfused paralleled increased TASH (Trauma-Associated Severe Hemorrhage) scores. Similarly, both fluid administration and the use of vasopressors increased through groups I to IV (Figure 1b).

\section{Validation of the new base deficit-based classification to the current ATLS classification of hypovolemic shock}

When the two approaches to classify the extent of hypovolemic shock upon ED admission were compared, the new BD-based classification displayed a higher accuracy for discriminating the need for early blood products than the current ATLS classification of hypovolemic shock (Figure 2). Through groups II to IV, the percentage of patients who had received at least 1 blood unit during early ED resuscitation was significantly higher compared with patients classified according to ATLS (Figure 2a). A similar pattern was noted for the frequency of MTs (Figure $2 \mathrm{~b}$ ). If patients were classified by BD, MT rates increased from $5 \%$ in class I (BD of not more than $2 \mathrm{mmol} / \mathrm{L}$ ) to $52 \%$ in class IV (BD of more than $10 \mathrm{mmol} / \mathrm{L}$ ). In contrast, when patients were classified according to ATLS, $4 \%$ of group I and only $25 \%$ of group IV patients received MT until ICU admission (Figure $2 \mathrm{~b}$ ). Furthermore, BD distinguished more precisely between patients at risk of dying than the current ATLS classification of hypovolemic shock (Figure 2c). If classified by BD, $7.4 \%$ of class I and $51.5 \%$ of class IV patients, on average, died during in-hospital stay. In contrast, patients classified according to ATLS showed mortality rates of $2 \%$ in class I and $31 \%$ in class IV patients.

\section{Discussion}

The aim of this study was to introduce and validate a new BD-based classification of hypovolemic shock for the initial assessment of trauma patients. This analysis was conducted on a cohort of not less than 16,305 severely injured patients derived from the TraumaRegister DGU $^{\mathbb{R}}$ database.

The early assessment of hypovolemic shock and the prediction of transfusion requirements in multiply 
Table 1 Patients classified by base deficit (classes I to IV): demographics, injury mechanism, severity of injury, and outcome

\begin{tabular}{|c|c|c|c|c|}
\hline & Class I & Class II & Class III & Class IV \\
\hline & $\begin{array}{l}\mathrm{BD} \leq 2.0 \\
\text { (no shock) }\end{array}$ & $\begin{array}{c}\mathrm{BD}>2.0 \text { to } 6.0 \\
\text { (mild) }\end{array}$ & $\begin{array}{c}\mathrm{BD}>6.0 \text { to } 10.0 \\
\text { (moderate) }\end{array}$ & $\begin{array}{c}\mathrm{BD}>10.0 \\
\text { (severe) }\end{array}$ \\
\hline \multicolumn{5}{|l|}{ Demographics } \\
\hline Total number (percentage) & $7,583(46.5)$ & $5,831(35.8)$ & $1,999(12.3)$ & $892(5.5)$ \\
\hline Male, number (percentage) & $5,622(74.7)$ & $4,184(72.3)$ & $1,382(69.6)$ & $607(68.4)$ \\
\hline Mean age (SD), years & $46(20.2)$ & $43.8(19.7)$ & $44.4(19.5)$ & $45.8(19.7)$ \\
\hline Blunt trauma, number (percentage) & $7,088(96)$ & $5,436(94.4)$ & $1,869(94.1)$ & $816(92.3)$ \\
\hline \multicolumn{5}{|l|}{ Injury severity } \\
\hline Mean ISS (SD), points & $19.1(11.9)$ & $24.0(13.3)$ & $29.5(16)$ & $36.7(17.6)$ \\
\hline Mean NISS (SD), points & $24.2(15)$ & $29.9(16.1)$ & $35.5(17.7)$ & $42.9(18.5)$ \\
\hline Mean RISC score (SD), points & $10.3(18.1)$ & $14.4(22.4)$ & $24.4(28.6)$ & $53.3(35.3)$ \\
\hline AlS head $\geq 3$ points, number (percentage) & $3,065(40.4)$ & $2,711(46.5)$ & $1,039(52)$ & $526(59)$ \\
\hline AIS thorax $\geq 3$ points, number (percentage) & $2,826(37.3)$ & $2,811(48.2)$ & $1,131(56.6)$ & $577(64.7)$ \\
\hline AIS abdomen $\geq 3$ points, number (percentage) & $819(10.8)$ & $939(16.1)$ & $520(26.0)$ & $296(33.2)$ \\
\hline AIS pelvis/extremities $\geq 3$ points, number (percentage) & $1,956(25.8)$ & $2,071(35.5)$ & $846(42.3)$ & $419(47.0)$ \\
\hline \multicolumn{5}{|l|}{ Outcome } \\
\hline Mortality, number (percentage) & $564(7.4)$ & $721(12.4)$ & $478(23.9)$ & $459(51.5)$ \\
\hline Mean hospital LOS (SD), days & $18,3(19.2)$ & $23.6(25.2)$ & $24.7(27)$ & $20.1(31.2)$ \\
\hline Mean ICU LOS (SD), days & $7.8(10.7)$ & $11.3(13.3)$ & $13.9(15.3)$ & $12.8(18.0)$ \\
\hline Mean ventilator days (SD) & $4.8(8.9)$ & $7.7(11.5)$ & $9.9(12.8)$ & $10.1(15.5)$ \\
\hline Multiple organ failure, number (percentage) & $807(12.2)$ & $1,064(20.2)$ & $516(29.4)$ & $294(43.3)$ \\
\hline Sepsis, number (percentage) & $400(6.0)$ & $566(10.5)$ & $295(16.3)$ & $126(18.0)$ \\
\hline
\end{tabular}

Cohort consisted of 16,305 patients. $P<0.001$ for all parameters. AIS, abbreviated injury scale; BD, base deficit; ICU, intensive care unit; ISS, injury severity score; LOS, length of stay; NISS, new injury severity score; RISC, Revised Injury Severity Classification; SD, standard deviation.

injured patients are still among the most challenging tasks in the initial management of trauma patients. One approach comprises the initial evaluation of vital signs as suggested by ATLS in its classification of hypovolemic shock by using combinations of HR, SBP, and GCS score. However, recent analyses on data of multiply injured patients derived from the TraumaRegister
$\mathrm{DGU}^{\circledR}$ and the TARN database indicated that the current ATLS classification of hypovolemic shock displays substantial deficits in allocating trauma patients into the corresponding classes $[3,4]$. Furthermore, the role of vital signs alone in the initial assessment of hypovolemic shock is still debated [3,15-18]. Paladino and colleagues [19] recently assessed the additional use of metabolic

Table 2 Patients classified by base deficit (classes I to IV): traditional vital signs as presented at emergency department admission and at the scene

\begin{tabular}{|c|c|c|c|c|}
\hline & Class I & Class II & Class III & Class IV \\
\hline & $\begin{array}{l}\mathrm{BD} \leq 2.0 \\
\text { (no shock) }\end{array}$ & $\begin{array}{c}\mathrm{BD}>2.0 \text { to } 6.0 \\
\text { (mild) }\end{array}$ & $\begin{array}{c}\mathrm{BD}>6.0 \text { to } 10.0 \\
\text { (moderate) }\end{array}$ & $\begin{array}{c}\mathrm{BD}>10.0 \\
\text { (severe) }\end{array}$ \\
\hline \multicolumn{5}{|l|}{ Vital signs } \\
\hline SBP at the scene, $\mathrm{mm} \mathrm{Hg}$ & $129.8(28.9)$ & $120.7(32.2)$ & $108.6(35.1)$ & $87.2(45.4)$ \\
\hline $\mathrm{SBP}$ at $\mathrm{ED}, \mathrm{mm} \mathrm{Hg}$ & $132.6(26.3)$ & $124.6(28.0)$ & $112.7(30.7)$ & $94.8(40.4)$ \\
\hline HR at the scene, beats per minute & $90.9(19.3)$ & $93.9(22.6)$ & $98.3(27)$ & $93.6(41)$ \\
\hline$H R$ at $E D$, beats per minute & $86.3(17.8)$ & $89.8(20.3)$ & $95.9(22.5)$ & $97.2(32.4)$ \\
\hline SI at the scene, beats per minute & $0.74(0.26)$ & $0.83(0.34)$ & $0.98(0.47)$ & $1.17(0.55)$ \\
\hline $\mathrm{SI}$ at ED, beats per minute & $0.68(0.22)$ & $0.76(0.27)$ & $0.93(0.41)$ & $1.17(0.69)$ \\
\hline Median GCS score at the scene (IQR), points & $14(10-15)$ & $13(6-15)$ & $10(3-15)$ & $4(3-12)$ \\
\hline Median GCS score at ED (IQR), points & $14(3-15)$ & $3(3-15)$ & $3(3-11)$ & $3(3-3)$ \\
\hline Intubation rate, number (percentage) & $2,732(40.2)$ & $3,319(60.5)$ & $1,417(73.9)$ & 724 (83.4) \\
\hline
\end{tabular}

Values are presented as mean (standard deviation) unless indicated otherwise. Cohort consisted of 16,305 patients. $P<0.001$ for all parameters. BD, base deficit; $\mathrm{ED}$, emergency department; GCS, Glasgow Coma Scale; HR, heart rate; IQR, interquartile range; SBP, systolic blood pressure; SI, shock index. 
Table 3 Patients classified by base deficit (classes I to IV): laboratory findings

\begin{tabular}{lcccc}
\hline & Class I & Class II & Class III & Class IV \\
\hline & $\begin{array}{c}\text { BD } \leq \mathbf{2 . 0} \\
\text { (no shock) }\end{array}$ & $\begin{array}{c}\text { BD }>\text { 2.0 to 6.0 } \\
\text { (mild) }\end{array}$ & $\begin{array}{c}\text { BD }>\text { 6.0 to 10.0 } \\
\text { (moderate) }\end{array}$ & $\begin{array}{c}\text { BD }>\text { 10.0 } \\
\text { (severe) }\end{array}$ \\
\hline Laboratory findings & & & & \\
\hline Hemoglobin, g/dL & $12.8(2.4)$ & $11.8(2.6)$ & $10.6(2.9)$ & $9.1(3.3)$ \\
Thrombocytes, tsd/ $\mu \mathrm{L}$ & $215(74)$ & $208(77)$ & $193(81)$ & $171(82)$ \\
Quick's value, percentage & $85.9(19.7)$ & $79.7(21.6)$ & $69.6(24)$ & $55.5(26.1)$ \\
aPTT, seconds & $29.8(9.2)$ & $32.1(13.7)$ & $39.0(23)$ & $69.5(41.1)$ \\
Lactate, mmol/L & $2.5(4.3)$ & $3.4(5.4)$ & $5.1(8.4)$ & $9.7(14)$ \\
\hline
\end{tabular}

Values are presented as mean (standard deviation). Cohort consisted of 16,305 patients. $P<0.001$ for all parameters. aPTT, activated partial thromboplastin time; $\mathrm{BD}$, base deficit.

parameters (for example, BD as a sensitive indicator of blood loss by measuring tissue perfusion) to traditional triage vital signs to distinguish major from minor trauma. In their retrospective single-center analysis, abnormal vital signs alone had a sensitivity of $40.9 \%$ for identifying major injury, but when abnormal metabolic parameters were added, the detection of major trauma increased significantly to a sensitivity of $76.4 \%$ [19].

In the present study, we propose a new classification based upon $\mathrm{BD}$, a parameter that indicates the presence of hypovolemic shock and identifies patients who are at risk to require blood product transfusions. In times of POCT, BD is available within minutes after ED admission. As early as 2005, Rixen and Siegel [9] suggested the evaluation of $\mathrm{BD}$ as a more useful approach to quantify the extent of hypovolemic shock than the estimation of blood loss, the extent of volume resuscitation, or vital signs such as HR and SBP. Additionally, these authors proclaimed that BD may be superior to the measurement of lactate levels.

The diagnostic use and prognostic value of $\mathrm{BD}$ are well documented. Out of 10 clinical and 20 laboratory parameters assessed, changes of BD have been proven to be the best predictor of blood volume change in a canine model of hemorrhagic shock [20]. On the basis of 1,810 multiply injured trauma patients derived from the TraumaRegister $D G U^{\circledR}$ database, potential predictors for transfusion requirements, including BD and lactate, have been identified via logistic regression. Seven variables could be identified to independently predict MT: gender (male), SBP, HR, hemoglobin, relevant injuries to the abdomen and extremities (Abbreviated Injury Scale score of at least 3), and BD, but not lactate $[21,22]$. Furthermore, our group has recently compared six scoring systems to predict the risk of ongoing hemorrhage and MT, including the TASH, Prince of Wales Hospital/Rainer (PWH/Rainer), Larson, Vandromme, Schreiber, and ABC (assessment of blood consumption) scores. The TASH and PWH/Rainer scores showed the highest overall accuracy in predicting ongoing hemorrhage and MT. Interestingly, both scores include BD as a laboratory surrogate for hypoperfusion. In contrast, only one scoring system (that is, the Vandromme score) comprises lactate [23]. Similarly, several mortality scores (for example, the Emergency Trauma Score (EMTRAS) [24] and BIG score [25]) use BD as the laboratory surrogate for shock. In the present study, worsening BD paralleled worsening lactate. However, the use of Ringer's lactate in the initial fluid resuscitation as well as the presence of ketoacidosis in patients with diabetes may influence lactate levels and can falsify the initial assessment $[9,26]$. The present study did not intend to address the question of whether BD or lactate may be superior in risk-stratifying trauma patients, and therefore this question remains unanswered. However, the data derived from the TraumaRegister $\mathrm{DGU}^{\circledR}$ database suggest that BD may be more accurate in detecting shock and blood loss as compared with lactate. Therefore, the proposed classification here is based on BD upon ED admission.

The present investigation revealed that increasing BD category reflected injury severity as demonstrated by an increasing injury severity score (ISS), new injury severity score (NISS), and RISC score and the incidence of MOF and sepsis. All of them are important factors influencing mortality and outcome of trauma patients. In our analysis, mortality rates rose from $7.4 \%$ to $51.5 \%$ with altered BD values. These observations are consistent with those of previous studies reporting an association between admission BD and mortality $[6,7,10,11]$. In a univariate logistic model, admission $\mathrm{BD}$ has been proven to be one of the best predictors for mortality, and a BD level of $6 \mathrm{mmol} / \mathrm{L}$ was identified as an important cutoff point for mortality $[7,11]$. Also, in pediatric and older trauma populations, BD has been shown to be an important indicator for injury severity and mortality [27-30]. Interestingly, the use of alcohol and drugs did not impair the predictive accuracy of admission BD with respect to trauma outcome [31].

In the present analysis, BD correlated with transfusion requirements, both in the overall amount of transfused 


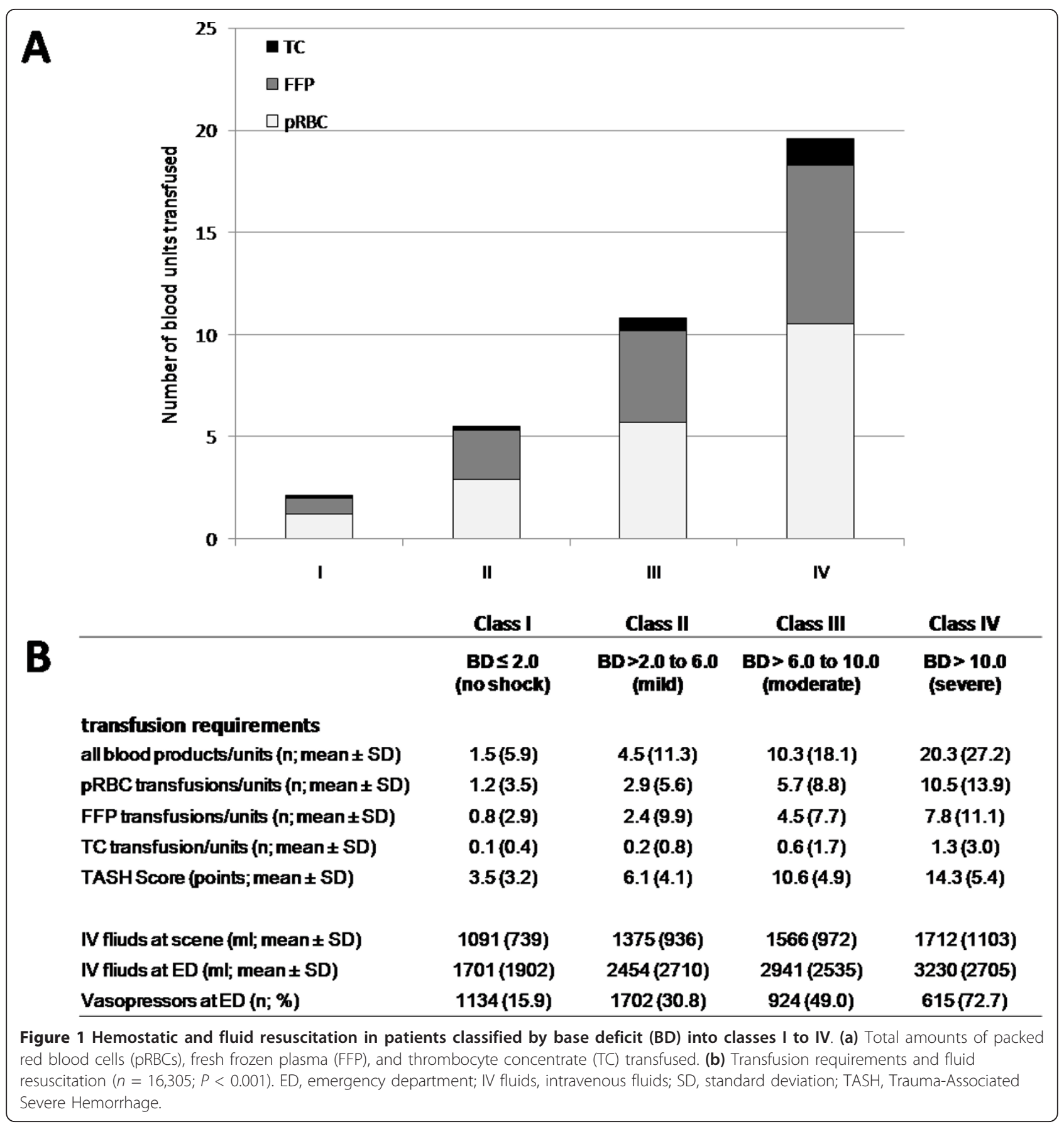

blood units and in the percentage of patients who required any blood transfusion ( $\geq 1$ blood unit). Furthermore, worsening BD paralleled increasing risk of ongoing hemorrhage as reflected by increasing TASH scores. The mean amount of blood products administered increased from $1.5 \pm 5.9$ to $20.3 \pm 27.2$ units with worsening BD category. These findings are consistent with those of a previous analysis demonstrating that worsening of $\mathrm{BD}$ was associated with an increased need for blood product transfusions $[6,7,32]$. Through the groups I to IV, the increasing amounts of intravenous fluids and vasopressors administered indicate the presence of hemodynamic instability and validated the results previously reported by Rixen and colleagues [7]. Laboratory findings such as decreases in hemoglobin levels and platelet counts and an impaired coagulation as reflected by a Quick's value of less than $70 \%$ were further interpreted as evidence for hypovolemic 


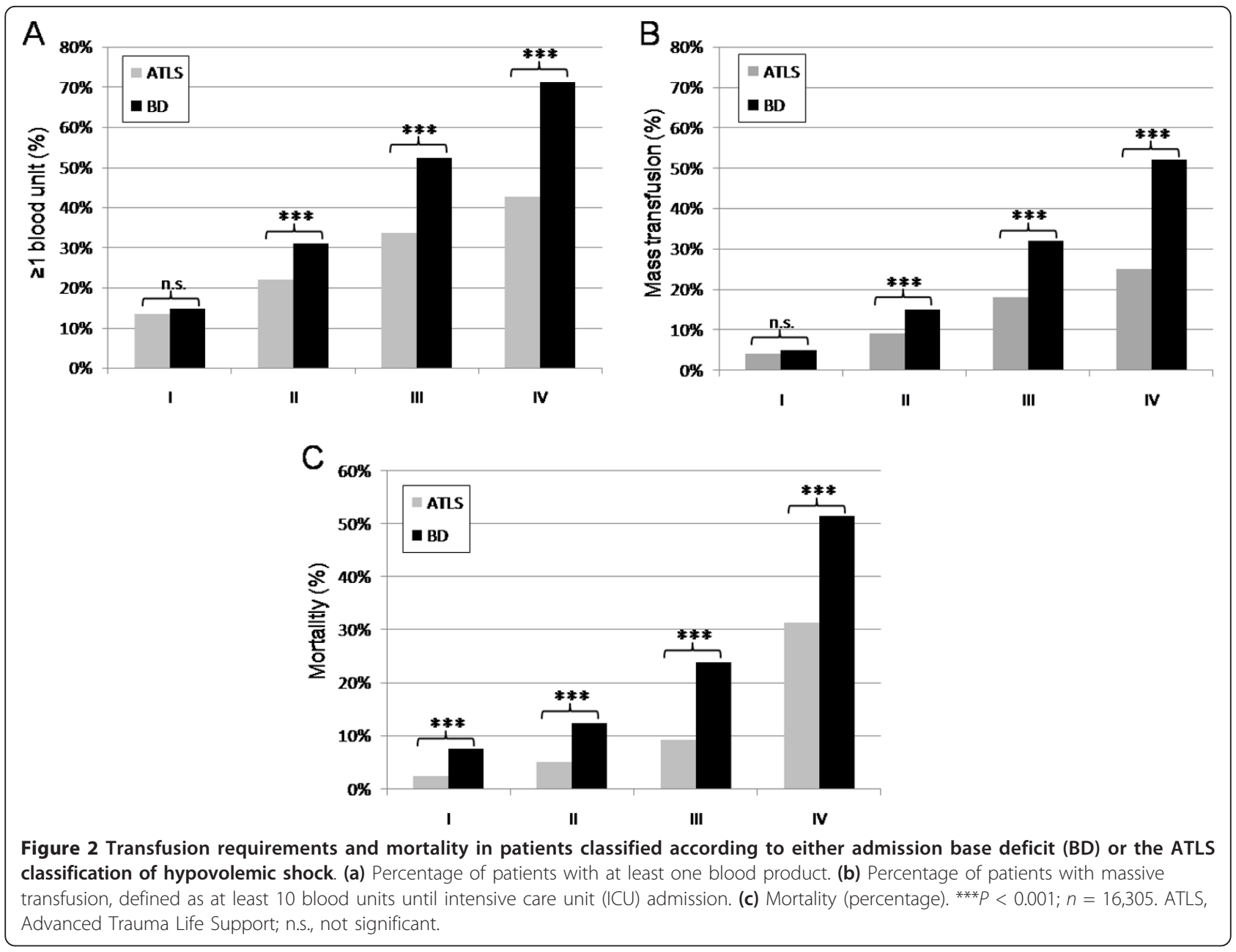

instability. Given these results, BD indicates the presence of hypovolemic shock related to hemostatic resuscitation need, transfusion requirements, laboratory findings, and mortality.

To the best of our knowledge, there is no gold standard to assess the presence of hypovolemic shock and to trigger therapeutic interventions. Thus, there is no option yet to test our novel approach against a gold standard. Therefore, the authors have decided to test against the current ATLS classification of hypovolemic shock given that this approach has been widely implemented in daily clinical routine as a standard protocol of care and for the initial assessment and treatment in trauma centers. Both the percentage of patients who had received at least one blood product and MTs were increased throughout the groups I to IV in both classifications. However, transfusion requirements were significantly higher when patients were classified by BD. Similar results were observed for mortality. Obviously, stratification by BD was associated with superior discrimination of trauma patients with respect to outcome and need for early blood products. In this context, ATLS seems to dramatically underestimate the need for blood product transfusion, particularly in group III and IV patients.

In summary, we suggest assessing patients in the ED on the basis of BD. Davis and colleagues [6] have already proposed that, in patients with a BD of less than $6 \mathrm{mmol} / \mathrm{L}$, blood typing should be sufficient but that patients with a BD of at least $6 \mathrm{mmol} / \mathrm{L}$ should undergo blood typing and cross-match. Given MT rates and the identification of patients who are in need of emergent transfusion, a BD of $6 \mathrm{mmol} / \mathrm{L}$ could also be suggested as a threshold. Table 4 displays our suggestion for a modified version of the current ATLS classification of hypovolemic shock based upon $\mathrm{BD}$ as a principal trigger for action. Following the ATLS paradigm of 'keep algorithms simple', specific recommendations are presented with regard to preparation and use of blood products. For class I and II patients, a careful observation should be sufficient unless clinical circumstances dictate otherwise. In class III patients, preparation for transfusion 
Table 4 A new base deficit-based classification of hypovolemic shock

\begin{tabular}{lcccc}
\hline & Class I & Class II & Class III & Class IV \\
\hline Shock & No shock & Mild & Moderate & Severe \\
\hline Base deficit at admission, $\mathrm{mmol} / \mathrm{L}$ & $\leq 2$ & $>2.0$ to 6.0 & $>6.0$ to 10.0 & $>10.0$ \\
\hline Need for blood products & Watch & Consider & Act & Be prepared for massive transfusion \\
\hline
\end{tabular}

should be initiated. In class IV patients, in whom MT rates were more than $50 \%$, the trauma leader should definitely be prepared for an MT (for example, by activation of an MT protocol and corresponding logistics).

The retrospective nature of this study and the modifications applied to the ATLS classification in order to conduct the present analysis are clear limitations of this study, and the authors are aware of this shortcoming. Although POCT can provide BD within minutes after ED admission, not every ED is equipped with this technology. However, ATLS claims that the knowledge and skills taught are easily adapted to all venues of trauma care. This implies that every ED worldwide as well as pre-hospital systems (Pre-hospital Trauma Life Support) use similar principles and assessment tools as suggested by ATLS. However, this study may be a first step toward a 'modified ATLS classification of hypovolemic shock' with improved clinical applicability. Further validation on other trauma databases and in prospective studies is needed, especially on cohorts including higher numbers of penetrating injuries. In the absence of POCT, future research is needed to develop alternative approaches (for example, modified and clinically adopted combinations of vital signs), which can be used as an equivalent to BD in the initial assessment of hypovolemic shock. Hereby, the basic and underlying ATLS concept focusing on its intentionally simple applicability, independent of venue, technical prerequisites, and time scales, would be preserved.

\section{Conclusions}

BD upon ED admission indicates the acute presence of hypovolemic shock related to the need for hemostatic resuscitation, transfusion, laboratory findings, and mortality. The four proposed classes of worsening BD seem to predict transfusion requirements and mortality more appropriately than the current ATLS classification of hypovolemic shock. BD might be a relevant clinical approach to early risk-stratify severely injured patients in the state of hypovolemic shock and for blood product transfusion during initial assessment.

\section{Key messages}

- The early recognition and management of hypovolemic shock remain among the most challenging tasks in the initial assessment of trauma patients.

- The current Advanced Trauma Life Support (ATLS) classification of hypovolemic shock displays deficits in reflecting clinical reality; therefore, we propose a new hypovolemic shock classification based on a metabolic marker sensitive to blood loss by measuring tissue perfusion (for example, base deficit (BD)

- A classification based on four groups of worsening BD correlates with the extent of hypovolemic shock in severely injured patients, as reflected by increased transfusion requirements, higher massive transfusion, and mortality rates.

- The new BD-based classification discriminates better the need for early blood product transfusion and mortality in severely injured patients than the current ATLS classification of hypovolemic shock.

\section{Abbreviations}

ATLS: Advanced Trauma Life Support; BD: base deficit; ED: emergency department; GCS: Glasgow Coma Scale; HR: heart rate; IMC: intermediate care; ICU: intensive care unit; MOF: multiple organ failure; MT: massive transfusion; POCT: point-of-care testing; PWH: Prince of Wales Hospital; RISC: revised injury severity classification; SBP: systolic blood pressure; SI: shock index; TARN: Trauma Audit and Research Network; TASH: Trauma-Associated Severe Hemorrhage.

\section{Authors' contributions}

MMu contributed to study design, acquisition and interpretation of data, and drafting of the manuscript. UN and BB contributed to analysis and interpretation of data and to revision of the manuscript. TB, AW, TF, and TP contributed to study design and to revision of the manuscript. MMae contributed to study conception and design, acquisition of data, analysis and interpretation of data, and revision of the manuscript. All authors read and approved the final manuscript.

\section{Competing interests}

The authors declare that they have no competing interests. This is an unfunded study.

\section{Author details}

${ }^{1}$ Department of Trauma and Orthopedic Surgery, Cologne-Merheim Medical Center (CMMC), University of Witten/Herdecke, Ostmerheimerstr. 200, D51109 Cologne, Germany. ${ }^{2}$ Institute for Research in Operative Medicine (IFOM), University of Witten/Herdecke, Ostmerheimerstr. 200, D-51109 Cologne, Germany. ${ }^{3}$ Academy for Trauma Surgery, Luisenstr. 58/59, D-10117 Berlin, Germany. ${ }^{4}$ Working Group on Polytrauma of the German Society for Trauma Surgery (DGU), Luisenstr. 58/59, D-10117 Berlin, Germany.

Received: 4 September 2012 Revised: 7 December 2012

Accepted: 11 January 2013 Published: 6 March 2013

\section{References}

1. American College of Surgeons, Committee on Trauma: Advanced Trauma Life Support for Doctors: Student Course Manual. 8 edition. Chicago: American College of Surgeons; 2008.

2. Kortbeek JB, Al Turki S, Ali J, Antoine J, Bouillon B, Brasel K, Brenneman F, Brink PR, Brohi K, Burris D, Burton R, Chapleau W, Cioffi W, Collet e Silva FDS, Cooper A, Cortes J, Eskesen V, Fildes J, Gautam S, Gruen RL, 
Gross R, Hansen KS, Henny W, Hollands MJ, Hunt RC, Jover Navalon JM, Kaufmann CR, Knudson P, Koestner A, Kosir R, et al: Advanced trauma life support, 8th edition, the evidence for change. J Trauma 2008, 64:1638-1650.

3. Mutschler M, Nienaber U, Brockamp T, Wafaisade A, Wyen H, Peiniger S, Paffrath T, Bouillon B, Maegele M: A critical reappraisal of the ATLS classification of hypovolaemic shock: Does it really reflect clinical reality? Resuscitation 2012.

4. Guly HR, Bouamra O, Little R, Dark P, Coats T, Driscoll P, Lecky FE: Testing the validity of the ATLS classification of hypovolaemic shock. Resuscitation 2010, 81:1142-1147.

5. Guly HR, Bouamra O, Spiers M, Dark P, Coats T, Lecky FE: Vital signs and estimated blood loss in patients with major trauma: testing the validity of the ATLS classification of hypovolaemic shock. Resuscitation 2011 82:556-559.

6. Davis JW, Parks SN, Kaups KL, Gladen HE, O'Donnell-Nicol S: Admission base deficit predicts transfusion requirements and risk of complications. J Trauma 1996, 41:769-774.

7. Rixen D, Raum M, Bouillon B, Lefering R, Neugebauer E: Base deficit development and its prognostic significance in posttrauma critical illness: an analysis by the trauma registry of the Deutsche Gesellschaft für Unfallchirurgie. Shock 2001, 15:83-89.

8. Rixen D, Siegel JH: Metabolic correlates of oxygen debt predict posttrauma early acute respiratory distress syndrome and the related cytokine response. J Trauma 2000, 49:392-403.

9. Rixen $\mathrm{D}$, Siegel $\mathrm{JH}$ : Bench-to-bedside review: oxygen debt and its metabolic correlates as quantifiers of the severity of hemorrhagic and post-traumatic shock. Crit Care 2005, 9:441-453.

10. Rutherford EJ, Morris JA, Reed GW, Hall KS: Base deficit stratifies mortality and determines therapy. J Trauma 1992, 33:417-423.

11. Siegel JH, Rivkind Al, Dalal S, Goodarzi S: Early physiologic predictors of injury severity and death in blunt multiple trauma. Arch Surg 1990, 125:498-508.

12. Trauma register of the German Society of Trauma Surgery. "Scoring" study committee of the German Society of Trauma Surgery. Unfallchirurgie 1994, 97:230-237, Article in German.

13. Wafaisade A, Lefering R, Tjardes $T$, Wutzler $S$, Simanski C, Paffrath $T$, Fischer P, Bouillon B, Maegele M, Trauma Registry of DGU: Acute coagulopathy in isolated blunt traumatic brain injury. Neurocrit Care 2010 12:211-219.

14. Wafaisade A, Wutzler $S$, Lefering R, Tjardes $T$, Banerjee M, Paffrath $T$, Bouillon B, Maegele M: Drivers of acute coagulopathy after severe trauma: a multivariate analysis of 1987 patients. Emerg Med J 2010, 27:934-939.

15. Brasel KJ, Guse C, Gentilello LM, Nirula R: Heart rate: is it truly a vital sign? J Trauma 2007, 62:812-817.

16. Rady MY, Smithline H, Blake H, Nowak R, Rivers E: A comparison of the shock index and conventional vital signs to identify acute, critical illness in the emergency department. Ann Emerg Med 1994, 24:685-690.

17. Jansen TC, Van Bommel J, Mulder PG, Rommes JH, Schieveld SJM, Bakker J: The prognostic value of blood lactate levels relative to that of vital signs in the pre-hospital setting: a pilot study. Crit Care 2008, 12:R160.

18. Parks JK, Elliott AC, Gentilello LM, Shafi S: Systemic hypotension is a late marker of shock after trauma: a validation study of Advanced Trauma Life Support principles in a large national sample. Am J Surg 2006, 192:727-731.

19. Paladino L, Sinert R, Wallace D, Anderson T, Yadav K, Zehtabchi S: The utility of base deficit and arterial lactate in differentiating major from minor injury in trauma patients with normal vital signs. Resuscitation 2008, 77:363-368.

20. Waisman Y, Eichacker PQ, Banks SM, Hoffman WD, MacVittie TJ, Natanson C: Acute hemorrhage in dogs: construction and validation of models to quantify blood loss. J Appl Physiol 1993, 74:510-519.

21. Yücel N, Lefering $R$, Maegele $M$, Vorweg M, Tjardes T, Ruchholtz $S$, Neugebauer EaM, Wappler F, Bouillon B, Rixen D: Trauma Associated Severe Hemorrhage (TASH)-Score: probability of mass transfusion as surrogate for life threatening hemorrhage after multiple trauma. $J$ Trauma 2006, 60:1228-1236, discussion 1236-1237.

22. Maegele M, Lefering $R$, Wafaisade A, Theodorou P, Wutzler S, Fischer $P$, Bouillon B, Paffrath T: Revalidation and update of the TASH-Score: a scoring system to predict the probability for massive transfusion as a surrogate for life-threatening haemorrhage after severe injury. Vox Sanguinis 2011, 100:231-238.

23. Brockamp $T$, Nienaber $U$, Mutschler $M$, Wafaisade A, Peiniger $S$, Lefering $R$, Bouillon B, Maegele M, Dgu T: Predicting on-going hemorrhage and transfusion requirement after severe trauma: a validation of six scoring systems and algorithms on the TraumaRegister DGU(R). Crit Care 2012, 16:R129.

24. Raum MR, Nijsten MW, Vogelzang M, Schuring F, Lefering R, Bouillon B, Rixen D, Neugebauer EA, Ten Duis HJ, Polytrauma Study Group of the German Trauma Society: Emergency trauma score: an instrument for early estimation of trauma severity. Crit Care Med 2009, 37:1972-1977.

25. Borgman MA, Maegele M, Wade CE, Blackbourne LH, Spinella PC: Pediatric trauma BIG score: predicting mortality in children after military and civilian trauma. Pediatrics 2011, 127:e892-897.

26. Raum M, Rixen D, Linker R, Gregor S, Holzgraefe B, Neugebauer E: Influence of lactate infusion solutions on the plasma lactate concentration. Anasthesiol Intensivmed Notfallmed Schmerzther 2002, 37:356-358, Article in German.

27. Jung J, Eo E, Ahn K, Cheon Y: Initial base deficit as predictors for mortality and transfusion requirement in the severe pediatric trauma except brain injury. Pediatr Emerg Care 2009, 25:579-581.

28. Kincaid EH, Chang MC, Letton RW, Chen JG, Meredith JW: Admission base deficit in pediatric trauma: a study using the National Trauma Data Bank. J Trauma 2001, 51:332-335.

29. Randolph LC, Takacs M, Davis KA: Resuscitation in the pediatric trauma population: admission base deficit remains an important prognostic indicator. J Trauma 2002, 53:838-842.

30. Davis JW, Kaups KL: Base deficit in the elderly: a marker of severe injury and death. J Trauma 1998, 45:873-877.

31. Dunne JR, Tracy JK, Scalea TM, Napolitano LM: Lactate and base deficit in trauma: Does alcohol or drug use impair their predictive accuracy? J Trauma 2005, 58:959-966

32. Tremblay LN, Feliciano DV, Rozycki GS: Assessment of initial base deficit as a predictor of outcome: mechanism of injury does make a difference. Am Surg 2002, 68:689-693, discussion 693-694.

\section{doi:10.1186/cc12555}

Cite this article as: Mutschler et al:: Renaissance of base deficit for the initial assessment of trauma patients: a base deficit-based classification for hypovolemic shock developed on data from 16,305 patients derived from the TraumaRegister DGU ${ }^{\mathbb{R}}$. Critical Care 2013 17:R42.

\section{Submit your next manuscript to BioMed Central and take full advantage of:}

- Convenient online submission

- Thorough peer review

- No space constraints or color figure charges

- Immediate publication on acceptance

- Inclusion in PubMed, CAS, Scopus and Google Scholar

- Research which is freely available for redistribution

Submit your manuscript at www.biomedcentral.com/submit
C Biomed Central 